\title{
The Impact of Ignoring Interval Censoring in Progression- Free Survival in Cancer Trials: A Systematic Review
}

\author{
Xiawen Zhang ${ }^{1}$, Eleanor Pullenayegum ${ }^{1,2}$ and Kelvin Kar-Wing Chan ${ }^{3,4}$ \\ ${ }^{1}$ Biostatistics Division, Dalla Lana School of Public Health, University of Toronto, Toronto, Canada \\ ${ }^{2}$ Child Health Evaluative Sciences, The Hospital for Sick Children, Toronto, Canada \\ ${ }^{3}$ Odette Cancer Research Program, Sunnybrook Research Institute, Toronto, Canada \\ ${ }^{4}$ Department of Medicine, University of Toronto, Toronto, Canada \\ E-mail: xiawen.zhang@mail.utoronto.ca
}

Received May 15, 2020

Accepted for publication June 1, 2020

Published online September 26, 2021

\begin{abstract}
Introduction: From statistical literature, the bias in treatment effect from ignoring interval censoring in Progression-free survival (PFS) is demonstrated. However, the impact on estimators caused by interval censoring is not carefully took account and investigated by researchers in practice. The objective of this study is to examine the impact of accounting for interval censoring in practice among RCTs used to support FDA approvals anti-cancer drugs between the years 2005 and 2019 that used PFS as an endpoint.
\end{abstract}

Methods: In this systematic review, the differences of hazard ratios between two methods: considering and ignoring interval censoring, are visualized by Kaplan-Meier survival curves and estimated from a Cox proportional hazard model of 87 RCTs. With assumption that these differences and mean differences (bias) follow a normal distribution, limits of agreement of differences and confidence interval of bias are used to represent agreement of two methods.

Results: Limits of agreement of difference range from -0.044 to 0.0615 , while confidence intervals for the bias range from 0.0026 to 0.0145 , which does not include zero, resulting in estimated treatment effect differs for two methods.

Conclusion: In general, bias caused by interval censoring in treatment effect exists with large sample studies. Focusing on individual clinical trials, limits of agreement can provide more information for researchers to make decision on how to account for interval censoring. 